\title{
Costs and Perioperative Outcomes Associated with Open versus Endoscopic Resection of Sinonasal Malignancies with Skull Base Involvement
}

Terence S. Fu ${ }^{1,2}$ Eric Monteiro ${ }^{2}$ Ian Witterick ${ }^{2}$ Allan Vescan ${ }^{2}$ Gelareh Zadeh $^{3}$ Fred Gentili ${ }^{3}$ John R. de Almeida ${ }^{2}$

1 Faculty of Medicine, University of Toronto, Toronto, Canada

${ }^{2}$ Department of Otolaryngology-Head \& Neck Surgery, University of

Toronto, Toronto, Canada

${ }^{3}$ Department of Neurosurgery, University of Toronto,

Toronto, Canada

Address for correspondence John R. de Almeida, MD, MSc, Department of Otolaryngology-Head \& Neck Surgery, University of Toronto, Princess Margaret Hospital, 610 University Avenue, 3-955, Toronto, ON, M5G 2M9, Canada (e-mail: john.dealmeida@uhn.ca).

J Neurol Surg B 2017;78:430-440.

\begin{abstract}
Objective To compare financial and perioperative outcomes between endoscopic and open surgical approaches in the surgical management of sinonasal malignancies.

Design Retrospective chart review.

Setting Tertiary care hospital.

Participants Patients undergoing surgical resection of a sinonasal malignancy from January 2000 to December 2014.

Main Outcome Measures In-hospital costs, complications, and length of stay (LOS). Results Of 106 patients, 91 received open surgery (19 free flap and 72 non-free flap) and 15 were treated with purely endoscopic approaches. Free flaps had a significantly higher average cost, operative time, and LOS compared to both non-free flap $(p<0.001,<0.001$, and $<0.01)$ and endoscopic $(p=0.01,0.04$, and $<0.01)$ groups. There were no significant differences in average costs between endoscopic and non-free flap groups ( $\$ 19,157$ vs. $\$ 14,806, p=0.20)$ or LOS (5.7 vs. 6.4 days, $p=0.72$ ). Compared with the non-free flap group, the endoscopic group had a longer average operative time ( 8.3 vs. 5.5 hours, $p<0.01$ ) and higher rates of cerebrospinal fluid (CSF) leak (13 vs. $0 \%, p=0.01$ ) and intensive care unit (ICU) admission (80 vs. $36 \%, p<0.01$ ). Surgical approach (open vs. endoscopic) was not a significant predictor

Keywords

- costs

- complications

- length of stay

- endoscopic surgery

- sinonasal malignancy of any financial or perioperative outcome on multivariable analysis.

Conclusion Hospital costs are comparable between endoscopic and open approaches when no free tissue reconstruction is required. Longer operative times, higher CSF leak rates, and our institutional protocol necessitating ICU admission for endoscopic cases may account for the failure to demonstrate cost savings with endoscopic surgery.
\end{abstract}

received

December 5, 2016

accepted after revision

May 12, 2017

published online

June 30, 2017 (c) 2017 Georg Thieme Verlag KG

Stuttgart · New York
Dol https://doi.org/

10.1055/s-0037-1603907. ISSN 2193-6331. 


\section{Introduction}

Recent decades have seen a paradigm shift in the surgical management of sinonasal and skull base tumors, as minimallyinvasive endoscopic approaches have been increasingly adopted as an alternative to traditional craniofacial resection (CFR). Although concerns existed initially regarding the oncological principles of endoscopic techniques, there is growing evidence supporting its safety and oncological effectiveness in carefully selected patients. ${ }^{1-3}$ Recent meta-analyses and large series comparing open and endoscopic approaches demonstrated comparable rates of long-term survival and locoregional control for a variety of sinonasal malignancies, including olfactory neuroblastomas, ${ }^{4,5}$ adenocarcinomas, ${ }^{6,7}$ squamous cell carcinomas, ${ }^{1,8}$ and malignant melanomas. ${ }^{2,9}$

Many malignancies that traditionally required an external approach can now be treated with endoscopic resection, resulting in less invasive surgery with lower mortality and morbidity, but comparable oncological outcomes. Recent studies have reported complication rates of 7 to $11 \%$ and a mortality of less than $1 \%$ for endoscopic surgery; $;$,2,5,7,10,11 in contrast, CFR is reported to have complication rates of 15 to $53 \%$ and a mortality of 5 to $13 \% .^{5,10-14}$ Additionally, studies have reported other favorable perioperative outcomes associated with endoscopic approaches, including reduced intraoperative blood loss, shorter hospital length of stay (LOS), and improved cosmesis. ${ }^{1,2,10,15}$

As endoscopic surgery emerges as a viable approach for skull base surgery, it has become increasingly important to delineate the costs and benefits to patients and their families. Furthermore, reporting on fiscal outcomes, such as costs, complications, and LOS, and identifying determinants of these outcomes, are critical toward guiding cost-effective health care utilization. Yet, there is a paucity of research describing the financial burden of surgical resection of sinonasal malignancies, ${ }^{16}$ and no studies have identified key factors impacting these outcomes.

The purpose of this study was to compare in-hospital costs and perioperative outcomes between open and endoscopic surgical approaches for sinonasal malignancies. A secondary aim was to identify patient- and treatment-related factors impacting these outcomes.

\section{Materials and Methods}

\section{Study Design}

This study included a retrospective chart review and financial data collection approved by the research ethics board at the University Health Network in Toronto, Ontario (no. 15-9623-CE). Data related to patient demographics, pathology, and treatment were obtained from electronic medical records, and case costing data were obtained from the finance department at the University Health Network. All adult patients (18 or older) undergoing skull base surgery for a sinonasal malignancy from January 2000 to December 2014 were reviewed. Patients with incomplete financial data, metastatic disease, or primary cutaneous malignancies with sinonasal tract extension were excluded.

\section{Data Extraction}

All clinical and operative notes were reviewed and data were recorded on patient demographics, tumor characteristics, and treatment details. Outcomes included in-hospital costs, LOS, length of operation, and complications occurring within a 30-day perioperative period. Comorbidity was graded using the Charlson comorbidity index (CCI), excluding the index cancer diagnosis from the solid tumor category. ${ }^{17}$

\section{Cost Data}

Financial data were obtained from the finance department at the University Health Network. The costs of care were defined as in-hospital costs related to the patient stay, and included all services involved in direct patient care: perioperative care, ICU, nursing, pharmacy, allied health (i.e., physical therapy, occupational therapy, speech language pathology, respiratory therapy, social work), and diagnostic laboratories and imaging. Overhead costs such as administration, equipment, finance, housekeeping, and human resources were also recorded, as were the costs of readmissions related to perioperative complications. Costs of care did not include physician fees, rehabilitation, or services provided after hospitalization. All costs were converted to 2015 Canadian dollars (CAD) using the historical Consumer Price Index (CPI) from Statistics Canada. ${ }^{18}$

\section{Statistical Analysis}

Descriptive statistics were used to describe the patient population and outcomes. Open free flap, non-free flap, and endoscopic groups were compared across patient, tumor, and treatment characteristics using analysis of variance (ANOVA) or chi-square test. Linear regression was used to identify predictors of in-hospital costs, complications, and LOS. Surgical approach (open vs. endoscopic) and other variables that were significant on univariable analysis were entered into a multiple regression model. All statistical analyses were conducted using SPSS version 21.0 (SPSS Inc., Chicago, Illinois, United States). A $p$-value of less than $5 \%$ was considered significant.

\section{Results}

During the 15-year period from January 2000 to December 2014, 164 adult patients underwent surgery for a sinonasal malignancy with skull base involvement at our institution. Of these, 57 were excluded due to incomplete financial data and 1 was excluded due to metastatic disease. The remaining 106 patients were eligible for inclusion.

\section{Patient, Tumor, and Treatment Characteristics}

Population characteristics are shown in -Table 1. Of 106 patients, 91 received open surgery (19 free flap and 72 nonfree flap) and 15 were treated with purely endoscopic approaches. Overall, 61 males and 45 females were treated. The median age was 56 years (range: 19-92 years). The most common histopathologies were squamous cell carcinoma, olfactory neuroblastoma, adenocarcinoma, malignant melanoma, and adenoid cystic carcinoma, which together accounted for 88 of 106 (83\%) patients. Eighty-five patients received radiotherapy and 15 received chemotherapy. Local and regional flap 
432 Costs of Malignant Sinonasal Tumor Resection Fu et al.

Table 1 Descriptive statistics

\begin{tabular}{|c|c|c|c|c|}
\hline Characteristic & Endoscopic $(n=15)$ & Open/Free flap $(n=19)$ & Open/Non-free Flap $(n=72)$ & $p$-Value ${ }^{a}$ \\
\hline Median age in years (range) & $53(25-73)$ & $56(23-76)$ & $57(23-90)$ & 0.62 \\
\hline \multicolumn{5}{|l|}{ Sex } \\
\hline Male & $9(60)$ & $11(58)$ & $41(57)$ & 0.88 \\
\hline Female & $6(40)$ & $8(42)$ & $31(43)$ & \\
\hline $\mathrm{CCl}($ mean $\pm \mathrm{SE})$ & $0.27 \pm 0.2$ & $1.4 \pm 0.3$ & $0.72 \pm 0.4$ & 0.04 \\
\hline \multicolumn{5}{|l|}{ Histopathology } \\
\hline Squamous cell carcinoma & $5(33)$ & $4(21)$ & $20(28)$ & 0.73 \\
\hline Olfactory neuroblastoma & $5(33)$ & $2(11)$ & $17(24)$ & \\
\hline Adenocarcinoma & $2(13)$ & $5(26)$ & $11(15)$ & \\
\hline Malignant melanoma & $0(0)$ & $1(5)$ & $8(11)$ & \\
\hline Adenoid cystic carcinoma & $1(7)$ & $3(16)$ & $4(6)$ & \\
\hline Mucoepidermoid carcinoma & $0(0)$ & $1(5)$ & $3(4)$ & \\
\hline Other $^{\mathrm{b}}$ & $2(13)$ & $3(16)$ & $9(13)$ & \\
\hline Recurrent & $14(93)$ & $7(37)$ & $12(17)$ & 0.06 \\
\hline \multicolumn{5}{|l|}{ Involvement } \\
\hline Orbit & $3(20)$ & $8(42)$ & $8(11)$ & $<0.01$ \\
\hline Dural & $6(40)$ & $5(26)$ & $19(26)$ & 0.55 \\
\hline Brain & $0(0)$ & $1(5)$ & $4(6)$ & 0.65 \\
\hline Palate & $0(0)$ & $5(26)$ & $7(10)$ & 0.06 \\
\hline Radiotherapy & $13(87)$ & $16(84)$ & $56(78)$ & 0.65 \\
\hline Chemotherapy & $5(33)$ & $2(11)$ & $8(11)$ & 0.07 \\
\hline \multicolumn{5}{|l|}{ Reconstruction } \\
\hline Local & $3(20)$ & $0(0)$ & $7(10)$ & 0.14 \\
\hline Regional & $1(7)$ & $4(21)$ & $23(32)$ & 0.11 \\
\hline Free flap & $0(0)$ & $19(100)$ & $0(0)$ & - \\
\hline Neck dissection & $0(0)$ & $6(32)$ & $7(10)$ & - \\
\hline Orbital exenteration & $0(0)$ & $6(32)$ & $0(0)$ & - \\
\hline
\end{tabular}

Abbreviations: $\mathrm{CCl}$, Charlson comorbidity index; SE, standard error.

a $p$-Value: Comparison to endoscopic group.

${ }^{b}$ Other histopathologies include hemangiopericytoma, neuroendocrine carcinoma, sinonasal undifferentiated carcinoma (SNUC), transitional cell papilloma, adult T-cell lymphoma, chondrosarcoma, mucosal lentiginous melanoma, myofibroblastic sarcoma, osteosarcoma, and plasmacytoma.

reconstruction were performed on 15 and 37 patients, respectively. Of the 91 patients undergoing open surgery, 13 underwent neck dissection and 6 underwent orbital exenteration.

Comparison between free flap, non-free flap, and endoscopic groups revealed no difference in age, sex, histopathology, dural/brain/palate involvement, or use of adjuvant radiation or chemotherapy (-Table $\mathbf{1}$ ). However, the free flap and non-free flap open surgery groups had higher average comorbidity levels $(p=0.04)$, and the non-free flap group had a lower proportion of patients with orbital involvement $(p<0.01)$.

\section{Outcomes for Open versus Endoscopic Surgery}

Average in-hospital costs were $\$ 28,770$ for free flap surgery, $\$ 14,806$ for open non-free flap surgery, and $\$ 19,157$ for endoscopic surgery (-Table 2). Free flap costs were significantly higher compared with both non-free flap and endoscopic groups ( $p<0.001$ and $p=0.01$, - Fig. 1 ). On average,

Table 2 Summary of primary outcomes

\begin{tabular}{|l|l|l|l|l|l|}
\hline Outcome (mean) & Endo $(\boldsymbol{n}=\mathbf{1 5})$ & Open/Free Flap $(\boldsymbol{n}=19)$ & $p$-Value & Open/Non-free Flap $(\boldsymbol{n}=72)$ & $p$-Value \\
\hline Costs $^{\mathrm{b}}$ & $\$ 19,157$ & $\$ 28,770$ & 0.01 & $\$ 14,806$ & 0.20 \\
\hline LOS (d) & 5.7 & 11.2 & $<0.01$ & 6.4 & 0.72 \\
\hline OR time (h) & 8.3 & 10.9 & 0.04 & 5.5 & $<0.01$ \\
\hline
\end{tabular}

${ }^{a} p$-Value: Comparison to endoscopic group.

${ }^{\mathrm{b}}$ All figures reported in 2015 Canadian dollars.

Abbreviations: LOS, length of stay; OR, operating room. 


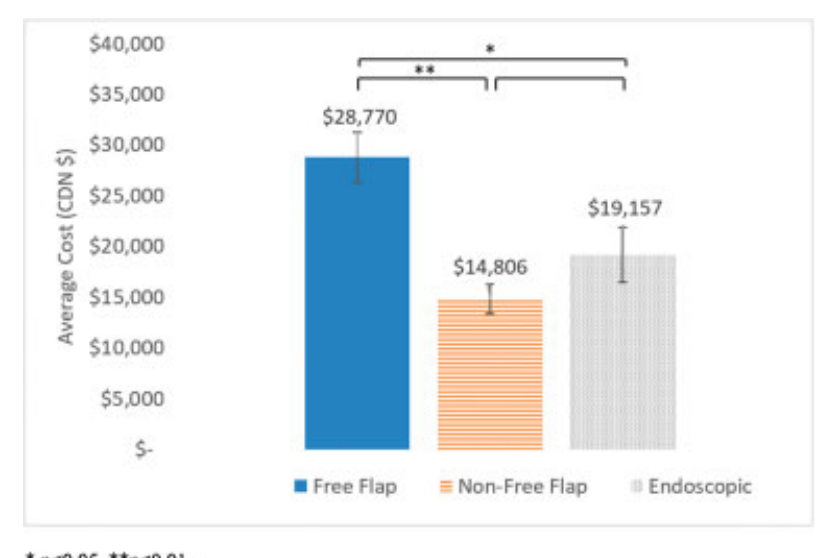

${ }^{*} \mathrm{p}<0.05,{ }^{* *} \mathrm{p}<0.01$.

Fig. 1 Average in-hospital cost associated with free flap, open non-free flap, and endoscopic surgery.

free flaps also required a significantly longer operative time (10.9 hours) compared with both non-free flap and endoscopic groups (5.5 and 8.3 hours, $p<0.01$ and $p=0.04$, respectively; -Fig. 2). Moreover, free flaps were associated with significantly longer LOS (11.2 days) compared with both non-free flap and endoscopic groups (6.4 and 5.7 days, $p<0.01$ for both, - Fig. 3 ).

While free flap surgery was clearly associated with higher costs, operative time, and LOS, differences between non-free flap and endoscopic surgery were less apparent. Compared with non-free flap patients, endoscopic surgery was not associated with an increased cost although there was a trend toward higher average cost $(\$ 19,157$ vs. $\$ 14,806$, $p=0.20 ;$ - Fig. 1). There was also no significant difference in average $\operatorname{LOS}$ ( 5.7 vs. 6.4 days, $p=0.72$; - Fig. 3). However, the endoscopic group did involve significantly longer operations on average (8.3 vs. 5.5 hours, $p<0.01$; - Fig. 2 ).

Perioperative complications included wound infection (4\%), CSF leak (2\%), hemorrhage (1\%), and intracranial abscess ( $1 \%$ ). There was no significant difference in overall complication rates between endoscopic and non-free flap groups ( $20 \mathrm{vs.} 8 \%$, $p=0.20 ;$-Table 3). However, the endoscopic group had

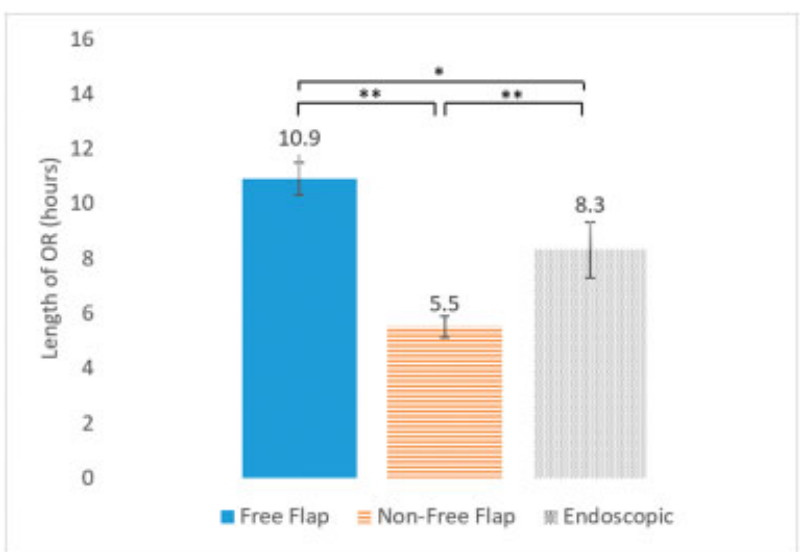

$* \mathrm{p}<0.05,{ }^{* *} \mathrm{p}<0.01$.

Fig. 2 Average operative time associated with free flap, open non-free flap, and endoscopic surgery.

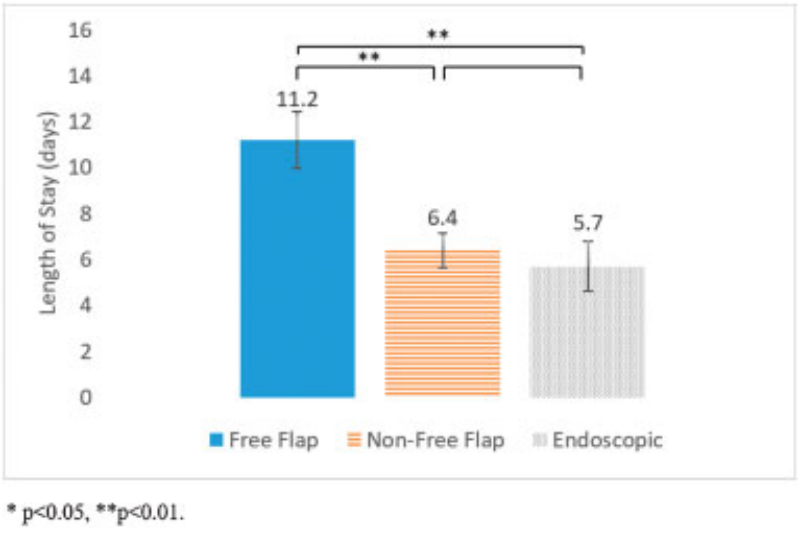

Fig. 3 Average hospital length of stay (LOS) associated with free flap, open non-free flap, and endoscopic surgery.

significantly higher rates of CSF leak (13 vs. $0 \%, p=0.01$ ) and ICU admission ( 80 vs. $36 \%, p<0.01$ ).

Further cost analysis by department as shown in - Fig. 4 demonstrated that patients undergoing endoscopic surgery incurred higher average perioperative costs $(\$ 11,037$ vs. $\$ 6,673, p<0.01)$ and ICU costs $(\$ 2,741$ vs. $\$ 1,688$, $p=0.02$ ) compared with non-free flap patients.

Table 3 Perioperative complications by surgical approach

\begin{tabular}{|l|l|l|l|l|}
\hline & $\begin{array}{l}\text { Endo } \\
(\boldsymbol{n}=15)\end{array}$ & $\begin{array}{l}\text { Open/Free } \\
\text { Flap } \\
(\boldsymbol{n}=19)\end{array}$ & $\begin{array}{l}\text { Open/Non-free } \\
\text { Flap }(\boldsymbol{n}=72)\end{array}$ & $p$-Value \\
\hline $\begin{array}{l}\text { Total } \\
\text { complications }\end{array}$ & $3(20)$ & $4(21)$ & $6(8)$ & 0.20 \\
\hline CSF leak & $2(13)$ & $1(5)$ & $0(0)$ & 0.01 \\
\hline ICU admission & $12(80)$ & $5(26)$ & $26(36)$ & $<0.01$ \\
\hline
\end{tabular}

Abbreviations: CSF, cerebrospinal fluid; ICU, intensive care unit. ${ }^{a}$-Value: Comparison to endoscopic group.

Abbreviations: CSF, cerebrospinal fluid; ICU, intensive care unit.

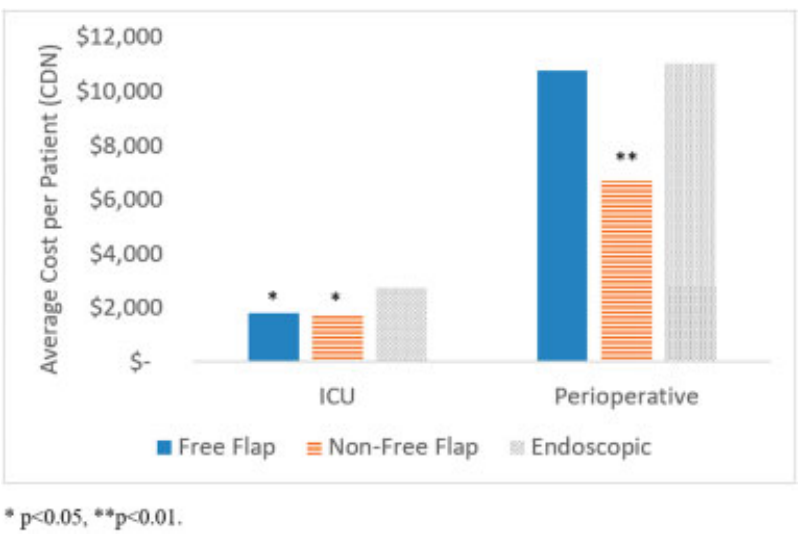

Fig. 4 Average per patient costs for intensive care unit (ICU) and perioperative care by surgical approach. 
434 Costs of Malignant Sinonasal Tumor Resection Fu et al.

\section{Predictors of In-Hospital Costs, Complications, and Length of Stay}

Factors associated with cost on univariable analysis were histopathology, orbital and dural involvement, preoperative radiation, regional and free flap reconstruction, orbital exenteration, perioperative complication, CSF leak, and ICU admission (-Table 4). However, on multivariable analysis, only free flap reconstruction, complication, and ICU admission were independently predictive of in-hospital $\operatorname{cost}(p<0.001$ for all).

Predictors of hospital LOS on univariable analysis included histopathology, regional and free flap reconstruction, perioperative complication, CSF leak, and ICU admission ( - Table 5). Again, however, only free flap reconstruction, complication, and ICU admission were independent predictors of LOS on multivariable analysis ( $p=0.001, p<0.001$, and $p=0.01$, respectively).

Finally, comorbidity level and regional and free flap reconstruction were associated with perioperative complications on univariable analysis. On multivariable analysis, however, only regional and free flap construction were significant predictors of complications ( $p=0.03$ and $p=0.04$; - Table 6 ).
Multiple regression revealed that surgical approach (open vs. endoscopic) was not a significant predictor of costs, LOS, or complications after controlling for relevant factors $(p=0.34$, $p=0.68$, and $p=0.25$, respectively; - Tables 4-6).

\section{Discussion}

Despite mounting pressure for cost containment on healthcare, there is a paucity of research on the costs of skull base surgery for sinonasal malignancies, and none that have directly compared costs between surgical approaches. Our study examined the in-hospital costs and perioperative outcomes of 106 patients undergoing skull base surgery for a sinonasal malignancy. The free flap group had a significantly higher average cost, operative time, and LOS compared with the other groups. There was no significant difference in LOS or costs between non-free flap and endoscopic surgery groups although there was a trend to higher costs in the endoscopic group. Multiple regression showed that free flap reconstruction was a significant predictor of in-hospital

Table 4 Predictors of in-hospital cost

\begin{tabular}{|l|l|l|l|l|}
\hline Characteristic & No of patients & Mean Cost $(\$)^{\text {a }}$ & $\begin{array}{l}\text { t-test } \\
(p \text {-value })\end{array}$ & $\begin{array}{l}\text { Multiple linear regression } \\
(p \text {-value })\end{array}$ \\
\hline Age & & & & \\
\hline$<65$ & 76 & 18,508 & 0.46 & \\
\hline$>65$ & 30 & 16,449 & & \\
\hline Sex & & & & \\
\hline Male & 61 & 18,175 & 0.82 & \\
\hline Female & 45 & 17,586 & & \\
\hline CCl & & & & \\
\hline 0 & 71 & 19,344 & 0.10 & \\
\hline$>1$ & 35 & 15,046 & & \\
\hline Histopathology & & & & \\
\hline Squamous cell carcinoma & 29 & 13,125 & 0.02 & 0.09 \\
\hline Olfactory neuroblastoma & 24 & 22,180 & & \\
\hline Adenocarcinoma & 18 & 19,333 & & \\
\hline Malignant melanoma & 9 & 14,336 & & \\
\hline Adenoid cystic carcinoma & 8 & 15,371 & & \\
\hline Mucoepidermoid carcinoma & 4 & 33,742 & & \\
\hline Other & 14 & 18,012 & & \\
\hline Recurrent & & & & \\
\hline No & 86 & 18,687 & & \\
\hline Yes & 20 & 14,648 & & \\
\hline Orbital & & 16,083 & & \\
\hline No & 19 & 26,360 & & \\
\hline Yes & 76 & & & \\
\hline Dural & 30 & 23,412 & & \\
\hline No & & & & \\
\hline Yes & & & \\
\hline
\end{tabular}


Costs of Malignant Sinonasal Tumor Resection Fu et al. 435

Table 4 (Continued)

\begin{tabular}{|c|c|c|c|c|}
\hline Characteristic & No of patients & Mean Cost $(\$)^{a}$ & $\begin{array}{l}\text { t-test } \\
\text { (p-value) }\end{array}$ & $\begin{array}{l}\text { Multiple linear regression } \\
\text { ( } p \text {-value) }\end{array}$ \\
\hline \multicolumn{5}{|l|}{ Brain } \\
\hline No & 101 & 17,747 & 0.52 & \\
\hline Yes & 5 & 21,521 & & \\
\hline \multicolumn{5}{|l|}{ Palate } \\
\hline No & 94 & 17,483 & 0.32 & \\
\hline Yes & 12 & 21,386 & & \\
\hline \multicolumn{5}{|l|}{ Radiotherapy } \\
\hline None & 21 & 13,336 & 0.04 & 0.23 \\
\hline Preoperative & 42 & 20,022 & & \\
\hline \multicolumn{5}{|l|}{ Chemotherapy } \\
\hline None & 90 & 17,866 & 0.70 & \\
\hline Preoperative & 14 & 19,316 & & \\
\hline \multicolumn{5}{|l|}{ Approach } \\
\hline Open/Combined & 91 & 17,722 & 0.69 & 0.43 \\
\hline Endoscopic & 15 & 19,157 & & \\
\hline \multicolumn{5}{|l|}{ Local flap } \\
\hline No & 96 & 18,122 & 0.63 & \\
\hline Yes & 10 & 16,031 & & \\
\hline \multicolumn{5}{|l|}{ Regional flap } \\
\hline No & 78 & 15,431 & $<0.001$ & 0.93 \\
\hline Yes & 28 & 24,873 & & \\
\hline \multicolumn{5}{|l|}{ Free flap } \\
\hline No & 87 & 15,556 & $<0.001$ & $<0.001$ \\
\hline Yes & 19 & 28,771 & & \\
\hline \multicolumn{5}{|l|}{ Neck dissection } \\
\hline No & 93 & 17,719 & 0.66 & \\
\hline Yes & 13 & 19,399 & & \\
\hline \multicolumn{5}{|l|}{ Orbital exenteration } \\
\hline No & 100 & 17,074 & $<0.01$ & 0.58 \\
\hline Yes & 6 & 32,101 & & \\
\hline \multicolumn{5}{|l|}{ Complication } \\
\hline No & 93 & 15,160 & $<0.001$ & $<0.001$ \\
\hline Yes & 13 & 37,706 & & \\
\hline \multicolumn{5}{|l|}{ CSF leak } \\
\hline No & 103 & 17,500 & 0.04 & \\
\hline Yes & 3 & 32,503 & & \\
\hline \multicolumn{5}{|l|}{ ICU stay } \\
\hline No & 63 & 11,588 & $<0.001$ & $<0.001$ \\
\hline Yes & 43 & 27,210 & & \\
\hline
\end{tabular}

Abbreviations: $\mathrm{CCl}$, Charlson comorbidity index; CSF, cerebrospinal fluid; ICU, intensive care unit.

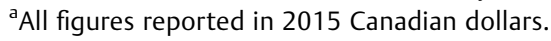


Table 5 Predictors of hospital LOS

\begin{tabular}{|c|c|c|c|c|}
\hline Characteristic & No. of patients & Mean LOS (d) & $\begin{array}{l}\text { t-test } \\
\text { (p-value) }\end{array}$ & Multiple linear regression ( $p$-value) \\
\hline \multicolumn{5}{|l|}{ Age } \\
\hline$<65$ & 76 & 6.1 & 0.25 & \\
\hline$<65$ & 30 & 7.3 & & \\
\hline \multicolumn{5}{|l|}{ Sex } \\
\hline Male & 61 & 6.8 & 9.86 & \\
\hline Female & 45 & 5.9 & & \\
\hline \multicolumn{5}{|l|}{$\mathrm{CCl}$} \\
\hline 0 & 71 & 6.4 & 0.46 & \\
\hline$<1$ & 35 & 6.5 & & \\
\hline \multicolumn{5}{|l|}{ Histopathology } \\
\hline Squamous cell carcinoma & 29 & 4.7 & 0.01 & 0.24 \\
\hline Olfactory neuroblastoma & 24 & 6.9 & & \\
\hline Adenocarcinoma & 18 & 6.8 & & \\
\hline Malignant melanoma & 9 & 5.4 & & \\
\hline Adenoid cystic carcinoma & 8 & 5.5 & & \\
\hline Mucoepidermoid carcinoma & 4 & 17.8 & & \\
\hline Other & 14 & 6.8 & & \\
\hline \multicolumn{5}{|l|}{ Recurrent } \\
\hline No & 86 & 6.3 & 0.86 & \\
\hline Yes & 20 & 6.9 & & \\
\hline \multicolumn{5}{|l|}{ Orbital } \\
\hline No & 87 & 6.2 & 0.20 & \\
\hline Yes & 19 & 7.4 & & \\
\hline \multicolumn{5}{|l|}{ Dural } \\
\hline No & 76 & 6.2 & 0.51 & \\
\hline Yes & 30 & 7.1 & & \\
\hline \multicolumn{5}{|l|}{ Brain } \\
\hline No & 101 & 6.5 & 0.53 & \\
\hline Yes & 5 & 5.4 & & \\
\hline \multicolumn{5}{|l|}{ Palate } \\
\hline No & 94 & 6.0 & 0.11 & \\
\hline Yes & 12 & 9.8 & & \\
\hline \multicolumn{5}{|l|}{ Radiotherapy } \\
\hline None & 21 & 5.9 & 0.59 & \\
\hline Preoperative & 42 & 6.8 & & \\
\hline \multicolumn{5}{|l|}{ Chemotherapy } \\
\hline None & 90 & 6.6 & 0.28 & \\
\hline Preoperative & 14 & 5.5 & & \\
\hline \multicolumn{5}{|l|}{ Approach } \\
\hline Open/Combined & 91 & 7.4 & 0.35 & 0.68 \\
\hline Endoscopic & 15 & 5.7 & & \\
\hline \multicolumn{5}{|l|}{ Local flap } \\
\hline No & 96 & 6.7 & 0.91 & \\
\hline
\end{tabular}


Table 5 (Continued)

\begin{tabular}{|c|l|l|l|l|}
\hline Characteristic & No. of patients & Mean LOS (d) & $\begin{array}{l}\text { t-test } \\
(p \text {-value })\end{array}$ & Multiple linear regression ( $p$-value) \\
\hline Yes & 10 & 3.7 & & \\
\hline Regional flap & & & & \\
\hline No & 78 & 5.7 & $<0.01$ & 0.23 \\
\hline Yes & 28 & 8.4 & & \\
\hline Free flap & & & & \\
\hline No & 87 & 5.6 & 0.001 & 0.001 \\
\hline Yes & 19 & 10.4 & & \\
\hline Neck dissection & & & & \\
\hline No & 93 & 5.9 & 0.08 & \\
\hline Yes & 13 & 10.0 & & \\
\hline Orbital exenteration & & & & \\
\hline No & 100 & 6.3 & 0.53 & \\
\hline Yes & 6 & 8.7 & & \\
\hline Complication & & & & \\
\hline No & 93 & 5.6 & $<0.001$ & $<0.001$ \\
\hline Yes & 13 & 12.5 & & \\
\hline CSF leak & & & & \\
\hline No & 103 & 6.4 & 0.04 & \\
\hline Yes & 3 & 8.0 & & \\
\hline ICU stay & 63 & 5.5 & $<0.01$ & 0.01 \\
\hline No & 43 & & \\
\hline Yes & & & \\
\hline
\end{tabular}

Abbreviations: CCl, Charlson comorbidity index; CSF, cerebrospinal fluid; ICU, intensive care unit; LOS, length of stay.

Table 6 Predictors of perioperative complications

\begin{tabular}{|l|l|l|l|l|}
\hline & No of patients & Complication, $\boldsymbol{n}(\%)$ & $\begin{array}{l}\text { t-test } \\
(p \text {-value })\end{array}$ & $\begin{array}{l}\text { Multiple linear regression } \\
(p \text {-value })\end{array}$ \\
\hline Characteristic & & & & \\
\hline Age & & & & \\
\hline$<65$ & 76 & $10(13)$ & 0.66 & \\
\hline$<65$ & 30 & $3(10)$ & & \\
\hline Sex & & & & \\
\hline Male & 61 & $8(13)$ & 0.76 & \\
\hline Female & 45 & $5(11)$ & & \\
\hline CCl & & & & 0.20 \\
\hline 0 & 71 & $12(17)$ & 0.04 & \\
\hline$<1$ & 35 & $1(3)$ & & \\
\hline Histopathology & & & & \\
\hline Squamous cell carcinoma & 29 & $2(7)$ & 0.77 & \\
\hline Olfactory neuroblastoma & 9 & $1(11)$ & & \\
\hline Adenocarcinoma & 24 & $4(17)$ & & \\
\hline Malignant melanoma & 18 & $3(17)$ & & \\
\hline Adenoid cystic carcinoma & 8 & $0(0)$ & & \\
\hline Mucoepidermoid carcinoma & 4 & $1(25)$ & & \\
\hline
\end{tabular}


Table 6 (Continued)

\begin{tabular}{|c|c|c|c|c|}
\hline & No of patients & Complication, $n$ (\%) & $\begin{array}{l}\text { t-test } \\
\text { ( } p \text {-value) }\end{array}$ & $\begin{array}{l}\text { Multiple linear regression } \\
\text { ( } p \text {-value) }\end{array}$ \\
\hline Other & 14 & & & \\
\hline \multicolumn{5}{|l|}{ Recurrent } \\
\hline No & 86 & $13(15)$ & 0.06 & \\
\hline Yes & 20 & $0(0)$ & & \\
\hline \multicolumn{5}{|l|}{ Orbital } \\
\hline No & 87 & $10(11)$ & 0.61 & \\
\hline Yes & 19 & $3(16)$ & & \\
\hline \multicolumn{5}{|l|}{ Dural } \\
\hline No & 76 & $8(11)$ & 0.39 & \\
\hline Yes & 30 & $5(17)$ & & \\
\hline \multicolumn{5}{|l|}{ Brain } \\
\hline No & 101 & $13(13)$ & 0.39 & \\
\hline Yes & 5 & $0(0)$ & & \\
\hline \multicolumn{5}{|l|}{ Palate } \\
\hline No & 94 & $12(13)$ & 0.66 & \\
\hline Yes & 12 & $1(8)$ & & \\
\hline \multicolumn{5}{|l|}{ Radiotherapy } \\
\hline None & 21 & $1(5)$ & 0.36 & \\
\hline Preoperative & 42 & $5(12)$ & & \\
\hline \multicolumn{5}{|l|}{ Chemotherapy } \\
\hline None & 90 & $12(13)$ & 0.52 & \\
\hline Preoperative & 14 & $1(7)$ & & \\
\hline \multicolumn{5}{|l|}{ Approach } \\
\hline Open/Combined & 91 & $10(11)$ & 0.32 & 0.25 \\
\hline Endoscopic & 15 & $3(20)$ & & \\
\hline \multicolumn{5}{|l|}{ Local flap } \\
\hline No & 96 & $10(10)$ & 0.07 & \\
\hline Yes & 10 & $3(30)$ & & \\
\hline \multicolumn{5}{|l|}{ Regional flap } \\
\hline No & 78 & $6(8)$ & 0.02 & 0.03 \\
\hline Yes & 28 & $7(25)$ & & \\
\hline \multicolumn{5}{|l|}{ Free flap } \\
\hline No & 87 & $9(10)$ & 0.02 & 0.04 \\
\hline Yes & 19 & $4(21)$ & & \\
\hline \multicolumn{5}{|l|}{ Neck dissection } \\
\hline No & 93 & $10(11)$ & 0.20 & \\
\hline Yes & 13 & $3(23)$ & & \\
\hline \multicolumn{5}{|l|}{ Orbital exenteration } \\
\hline No & 100 & $12(12)$ & 0.74 & \\
\hline Yes & 6 & $1(17)$ & & \\
\hline
\end{tabular}

Abbreviations: $\mathrm{CCl}$, Charlson comorbidity index; CSF, cerebrospinal fluid; ICU, intensive care unit.

costs, complications, and LOS. Complications and ICU admission were also independently predictive of costs and LOS. This is the first study, to our knowledge, to identify predictors of the costs and perioperative outcomes of skull base surgery.

To our knowledge, there is only one study reporting on the costs of skull base surgery. Stapelton et al ${ }^{16}$ conducted a retrospective review of 55 pediatric patients undergoing endoscopic surgery for a skull base lesion. This 2015 study reported an average in-hospital cost of \$34,056 USD with an average LOS of 4.5 days. In our series, the average cost of endoscopic surgery was $\$ 19,157 \mathrm{CDN}$ and average LOS was 5.7 days. Differences in patient demographics, geographic location, healthcare systems, time horizon, and tumor histopathology could explain the discrepancy compared with the costs in our study. 
In our study, free flap surgery was associated with significantly higher costs, operative time, and LOS compared with both non-free flap ( $p<0.001, p<0.01$, and $p<0.01$ ) and endoscopic approaches $(p=0.01, p=0.04, p<0.01)$. Free flaps also had the highest overall rate of complications among the three groups ( 21 vs. $20 \%$ endoscopy vs. $8 \%$ non-free flap, $p=0.20$ ). These results were consistent with multiple regression analysis, which showed that free flap reconstruction was a significant predictor of costs, complications, and hospital LOS.

Comparison between non-free flap and endoscopic groups was more challenging and warranted further discussion. In terms of complications, our study found a higher CSF leak rate in the endoscopic group (13 vs. $0 \%$ non-free flap, $p=0.01$ ) although there were no significant differences in overall complications $(p=0.20)$. These rates are comparable to studies in the literature, which report overall complication rates ranging from 3 to $26 \%$ for endoscopic surgery ${ }^{1,2,5,7,10,11}$ and 15 to $53 \%$ for open surgery. ${ }^{5,10-14}$ CSF leak rates in the literature are 3 to $12 \%$ for endoscopic surgery ${ }^{1,2,5,11}$ and 4 to $10 \%$ for open surgery. $1,5,11$

Our study failed to show a significant difference in the average LOS between endoscopic and non-free flap groups ( 5.7 vs. 6.4 days, $p=0.72$ ). The literature supports a shorter average LOS for patients undergoing an endoscopic approach ranging from 3.0 to 4.7 days ${ }^{1,2,5,7,10,11}$ compared with 5.7 to 11.5 days for those receiving open surgery. ${ }^{5,10-14}$ In one study, Nicolai et al $^{6}$ described a series of 67 patients with ethmoid adenocarcinoma treated purely with endoscopic surgery $(n=29)$, CFR $(n=18)$, and cranioendoscopic resection (CER) combining an endoscopic approach with subfrontal craniotomy $(n=9)$. This study found that CER and CFR increased hospital LOS by an average of 6.3 and 11.7 days, respectively, compared with purely endoscopic approaches. One major caveat is the need for free tissue reconstruction. As evidenced in our study, patients undergoing reconstructive procedures require longer hospital stay and this may account for some of the studies demonstrating improvements in LOS.

A common limitation of the aforementioned studies comparing surgical approaches is the lack of control for disparities in histopathology and tumor stage between open and endoscopic cohorts. In many studies, the endoscopic group contained less advanced tumors and prognostically better histopathologies such as olfactory neuroblastoma and adenocarcinoma compared with the open group. ${ }^{1,6,10,11}$ In our study, there were no significant differences in histopathology or markers of tumor severity such as dural/brain/palate involvement, apart from higher orbital involvement in the endoscopic group (20 vs. $11 \%$, $p<0.01)$. Multivariable analysis showed that surgical approach was not independently predictive of perioperative complication or LOS after controlling for relevant factors.

There was no statistical difference in costs between the endoscopic group and the open non-free flap group, $(\$ 19,157$ vs. $14,806, p=0.20$ ) although there was a trend toward increased costs for endoscopic patients. We hypothesize that these differences are primarily attributable to the significantly longer operating rooms (ORs) in the endoscopic group (8.3 vs. 5.5 hours, $p<0.01$ ). This is consistent with the results of our cost analysis by the department, which showed significantly higher perioperative costs for endoscopic patients compared with non-free flap patients ( $\$ 11,037$ vs. $\$ 6,673, p<0.01$ ). Other potential explanations could include the significantly higher rates of CSF leak and ICU admission in the endoscopic cohort. This is consistent with our multiple regression analysis, which identified perioperative complications and ICU admissions as independent predictors of increased costs. Although we did not delve into the reasons for ICU admission for this particular study, our institutional approach in general is to transfer patients undergoing an endoscopic approach to the ICU. Patients undergoing open approaches on the other hand do not necessarily require ICU admission. This suggests one potential cost-saving measure of transferring endoscopic patients to a step down unit or directly to the ward in the postoperative period.

Endoscopic skull base surgery requires a range of specialized instrumentation, which involves considerable cost, including videoendoscopic navigation, endoscopes, microdebriders, hemostasis systems, and disposable items such as microdebrider blades. ${ }^{19}$ Comparison to costs reported in the literature is difficult owing to a paucity of research in this area; this highlights the need for additional studies that evaluate the financial burden of evolving endoscopic approaches compared with traditional open surgery. ${ }^{16}$

This study is limited by the retrospective design, singleinstitution bias, and small sample size, particularly for the cohort of endoscopically treated patients. Our study did not include the costs of physician services, rehabilitation, or additional services provided after inpatient hospital stay. Additionally, we did not account for quality of life lost, productivity losses borne by family members, or intangible costs such as grief and pain; these costs, while difficult to measure, are likely to be substantial. Despite the above limitations, our study is the first to (1) compare the in-hospital costs of open and endoscopic surgical approaches for sinonasal malignancies, and (2) identify predictors of cost, complications, and hospital LOS among these patients. Larger, multi-institutional studies with more patients are needed to better evaluate the financial burden associated with sinonasal and skull base surgery, and confirm the findings of this study. Future studies could also examine the impact of surgical approach on quality of life and incorporate financial data on out-of-hospital costs in the postoperative period.

\section{Authors' Contributions}

All authors contributed extensively to the work presented in this paper. T.S.F. and J.R.A. jointly conceived the study design; T.S.F. collected and analyzed the data; T.S.F. interpreted the results and prepared the manuscript under supervision from E.M., I.W., A.V., and J.R.A.; E.M., I.W., A.V., G.Z., F.G., and J.R.A provided technical support and conceptual advice; all authors discussed the results and implications and edited the manuscript.

\section{Conflict of Interests}

The authors have no conflicts to disclose.

\section{Funding}

None declared. 


\section{Note}

The study was presented at the North American Skull Base Association at the 27th Annual Meeting in New Orleans, Louisiana on March 5, 2017.

\section{Acknowledgments}

We would like to thank Colleen Simpson, Jane Chen, Hongwei Ding, and Elizabeth Dos Santos at the University Health Network in Toronto, Ontario for their valuable administrative support and assistance in gathering important data for this study. Authors Terence Fu and John de Almeida had full access to all the data in the study and took responsibility for the integrity of the data and accuracy of the data analysis.

\section{References}

1 Hanna E, DeMonte F, Ibrahim S, Roberts D, Levine N, Kupferman M. Endoscopic resection of sinonasal cancers with and without craniotomy: oncologic results. Arch Otolaryngol Head Neck Surg 2009;135(12):1219-1224

2 Nicolai P, Battaglia P, Bignami M, et al. Endoscopic surgery for malignant tumors of the sinonasal tract and adjacent skull base: a 10-year experience. Am J Rhinol 2008;22(03):308-316

3 Wellman BJ, Traynelis VC, McCulloch TM, Funk GF, Menezes AH, Hoffman HT. Midline anterior craniofacial approach for malignancy: results of en bloc versus piecemeal resections. Skull Base Surg 1999;9(01):41-46

4 Devaiah AK, Andreoli MT. Treatment of esthesioneuroblastoma: a 16-year meta-analysis of 361 patients. Laryngoscope 2009; 119(07):1412-1416

5 Fu TS, Monteiro E, Muhanna N, Goldstein DP, de Almeida JR. Comparison of outcomes for open versus endoscopic approaches for olfactory neuroblastoma: a systematic review and individual participant data meta-analysis. Head Neck 2016;38(Suppl 1): E2306-E2316

6 Nicolai P, Villaret AB, Bottazzoli M, Rossi E, Valsecchi MG. Ethmoid adenocarcinoma-from craniofacial to endoscopic resections: a single-institution experience over 25 years. Otolaryngol Head Neck Surg 2011;145(02):330-337

7 Meccariello G, Deganello A, Choussy O, et al. Endoscopic nasal versus open approach for the management of sinonasal adeno- carcinoma: a pooled-analysis of 1826 patients. Head Neck 2016; 38(Suppl 1):E2267-E2274

8 de Almeida JR, Su SY, Koutourousiou M, et al. Endonasal endoscopic surgery for squamous cell carcinoma of the sinonasal cavities and skull base: oncologic outcomes based on treatment strategy and tumor etiology. Head Neck 2015;37(08):1163-1169

9 Moreno MA, Roberts DB, Kupferman ME, et al. Mucosal melanoma of the nose and paranasal sinuses, a contemporary experience from the M. D. Anderson Cancer Center. Cancer 2010;116(09): 2215-2223

10 Farquhar D, Kim L, Worrall D, et al. Propensity score analysis of endoscopic and open approaches to malignant paranasal and anterior skull base tumor outcomes. Laryngoscope 2016;126(08): 1724-1729

11 Eloy JA, Vivero RJ, Hoang K, et al. Comparison of transnasal endoscopic and open craniofacial resection for malignant tumors of the anterior skull base. Laryngoscope 2009;119(05): 834-840

12 Patel SG, Singh B, Stambuk HE, et al. Craniofacial surgery for esthesioneuroblastoma: report of an international collaborative study. J Neurol Surg B Skull Base 2012;73(03):208-220

13 Ganly I, Patel SG, Singh B, et al. Complications of craniofacial resection for malignant tumors of the skull base: report of an international collaborative study. Head Neck 2005;27(06):445-451

14 Ganly I, Patel SG, Singh B, et al. Craniofacial resection for malignant paranasal sinus tumors: report of an international collaborative study. Head Neck 2005;27(07):575-584

15 Castelnuovo P, Turri-Zanoni M, Battaglia P, Antognoni P, Bossi P, Locatelli D. Sinonasal malignancies of anterior skull base: histology-driven treatment strategies. Otolaryngol Clin North Am 2016;49(01):183-200

16 Stapleton AL, Tyler-Kabara EC, Gardner PA, Snyderman CH. The costs of skull base surgery in the pediatric population. J Neurol Surg B Skull Base 2015;76(01):39-42

17 Charlson ME, Pompei P, Ales KL, MacKenzie CR. A new method of classifying prognostic comorbidity in longitudinal studies: development and validation. J Chronic Dis 1987;40(05):373-383

18 Statistics Canada. Consumer Price Index, historical summary, 1996 to 2015 (CANSIM, table 326-0021 and Catalogue nos. 62001-X, 62-010-X and 62-557-X). Ottawa, ON: Statistics Canada; 2016

19 Verillaud B, Bresson D, Sauvaget E, et al. Endoscopic endonasal skull base surgery. Eur Ann Otorhinolaryngol Head Neck Dis 2012;129(04):190-196 